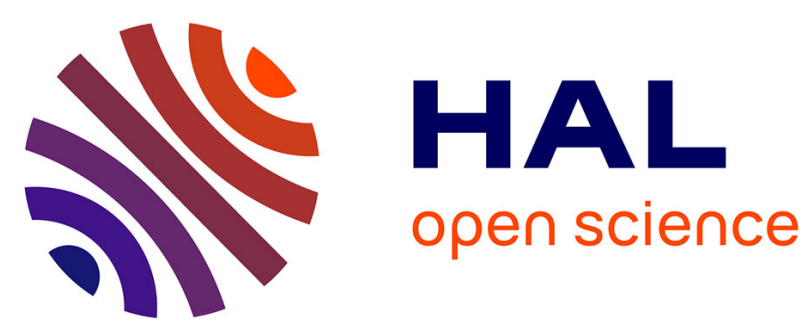

\title{
LE RÔLE DES AGGLOMÉRATS D'INTERSTITIELS DANS LA FORMATION DES DÉFAUTS DE FRENKEL DANS LES HALOGÉNURES ALCALINS
}

\author{
G. Guillot, P. Pinard, F. Herrmann
}

\section{- To cite this version:}

G. Guillot, P. Pinard, F. Herrmann. LE RÔLE DES AGGLOMÉRATS D'INTERSTITIELS DANS LA FORMATION DES DÉFAUTS DE FRENKEL DANS LES HALOGÉNURES ALCALINS. Journal de Physique Colloques, 1973, 34 (C9), pp.C9-113-C9-116. 10.1051/jphyscol:1973919 . jpa-00215394

HAL Id: jpa-00215394

https://hal.science/jpa-00215394

Submitted on 1 Jan 1973

HAL is a multi-disciplinary open access archive for the deposit and dissemination of scientific research documents, whether they are published or not. The documents may come from teaching and research institutions in France or abroad, or from public or private research centers.
L'archive ouverte pluridisciplinaire HAL, est destinée au dépôt et à la diffusion de documents scientifiques de niveau recherche, publiés ou non, émanant des établissements d'enseignement et de recherche français ou étrangers, des laboratoires publics ou privés. 


\title{
LE RÔLE DES AGGLOMÉRATS D'INTERSTITIELS DANS LA FORMATION DES DÉFAUTS DE FRENKEL DANS LES HALOGÉNURES ALCALINS
}

\author{
G. GUILLOT, P. PINARD \\ Laboratoire de Physique de la Matière, INSA, \\ 69621 Villeurbanne, France \\ F. HERRMANN \\ Institut für Angewandte Physik, Universität, \\ 75 Karlsruhe, Germany
}

\begin{abstract}
Résumé. - La concentration en centres $\mathrm{F}$ dans $\mathrm{KBr}$ et $\mathrm{KCl}$ sous irradiation électronique à la température de l'azote liquide évolue comme $t^{0,75}$ pour des cristaux purs et comme $t^{0,5}$ pour des cristaux dopés ou impurs. La discussion de ces résultats, à l'aide d'un modèle selon lequel la stabilisation des interstitiels détermine le taux de croissance, conduit aux conclusions suivantes :

1) La probabilité de capture d'un interstitiel par un piège est fonction de la taille du piège dans les cristaux purs:

2) La probabilité de capture est indépendante de la taille dans les cristaux impurs. Ceci s'explique en supposant une interaction à longue distance entre l'interstitiel et le piège.
\end{abstract}

Abstract. - The $\mathrm{F}$ centres concentration in $\mathrm{KBr}$ and $\mathrm{KCl}$ grows under electron irradiation at LNT as $t^{0.75}$ in pure and as $t^{0.5}$ in doped or impur crystals. The discussion of these findings, using a model according to which the growth rate is determined by interstitial stabilisation, results in the following conclusions :

1) The interstitial capture probability by a trap varies with the trap size in the pure crystals;

2) The capture probability is independent of the trap size in the impure samples. This can be explained by a long range interaction between traps and interstitials.

Il est maintenant bien établi que, dans les halogénures alcalins, l'irradiation avec des particules ionisantes crée des paires de Frenkel [1] suivant le modèle de Pooley [2] : un exciton se recombine non radiativement et fournit l'énergie et l'impulsion nécessaires à leur création. Une question importante se pose alors : que se passe-t-il après le processus primaire de création puisque les centres $F$ ne peuvent subsister que si les interstitiels sont stabilisés dans le réseau?

Un certain nombre de travaux ont montré en particulier que :

- à $80 \mathrm{~K}$, la présence d'impuretés augmente le rendement de création des centres $F$ [3] et modifie même les dommages de l'irradiation (déplacement des bandes $\mathrm{V}$ et réduction de la stabilité des défauts créés) [4], [5] ;

- à $300 \mathrm{~K}$, les interstitiels forment des agglomérats dont la taille peut être de l'ordre d'une centaine d'atomes d'halogènes [6], [7], [8], l'existence expérimentale de ces agglomérats étant moins nette à $80 \mathrm{~K}$.

Ces différents résultats sont expliqués par la présence, après l'acte primaire de création, de réactions secondaires au cours desquelles les interstitiels mobiles soit se recombinent avec les lacunes, soit se stabilisent sur des pièges à l'origine desquels se trouvent certainement des impuretés [9], [10].

Toutefois, un certain nombre de points obscurs demeurent; en particulier, une étude théorique à partir d'équations de taux montre que dans les échantillons très purs, on s'attend à trouver une loi de croissance des centres $F$ en racine carrée de la dose. Une telle loi a été trouvée dans LiF [10] mais les expériences de Sonder, avec $\mathrm{KCl}$, ont conduit à une loi quasi linéaire [9].

C'est pourquoi nous avons entrepris une étude expérimentale systématique de la loi de croissance des centres $F$ dans différents halogénures alcalins sous bombardement électronique, et envisagé des possibilités d'interprétation quantitative des résultats obtenus. La température de l'azote liquide a été choisie car, pour pouvoir appliquer le modèle cité [10], il est nécessaire que les interstitiels soient mobiles et que les lacunes et les centres $V_{k}$ soient fixes [II]. Dans ces conditions, on s'attend à avoir une description plus simple des cinétiques de création de défauts qu’à température ambiantc par excmple.

1. Résultats expérimentaux. - Nous présentons les résultats relatifs à $\mathrm{KBr}$ ct $\mathrm{KCl}$. Toutefois, nous 
nous sommes plus particulièrement attachés à $\mathrm{KBr}$, $\mathrm{KCl}$ semblant se comporter de façon identique.

Les échantillons sont irradiés à la température de l'azote liquide avec des électrons de moyenne énergie ( 5 à $50 \mathrm{keV}$ ) et des densités de courant $j$ variables entre 0,5 et $10 \mu \mathrm{A} / \mathrm{cm}^{2}$. La cinétique de croissance est enregistrée par absorption optique sous irradiation électronique dont l'avantage principal est de permettre d'atteindre des concentrations en centres $F$ élevées (quelque $10^{19} / \mathrm{cm}^{3}$ ) en des temps de bombardement relativement courts (quelques dizaines de minutes) et avec des densités optiques bien mesurables.

Nous avons irradié, dans les mêmes conditions, des cristaux de différentes provenances, en particulier des cristaux de Harshaw et Korth ultra-purs ainsi que des cristaux Harshaw dopés au moyen de diverses impuretés monovalentes et bivalentes. Nous n'avons pas fait d'analyses systématiques des impuretés présentes dans les cristaux; toutefois, il est à peu près certain [12] que ceux de provenance Harshaw sont plus purs que les cristaux Korth.

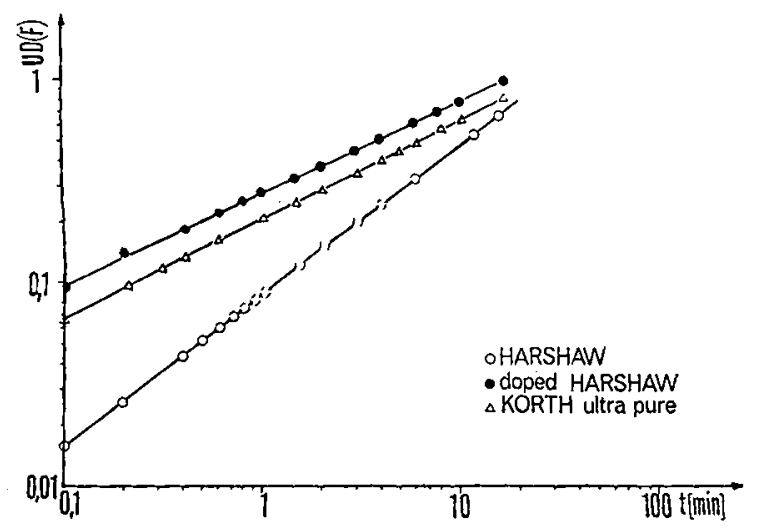

FIG. 1. - Cinétiques de croissance des centres $\mathrm{F}$ dans $\mathrm{KBr}$ de différentes origines à $80 \mathrm{~K}$ (irradiations à $V=20 \mathrm{kV}$, $\left.j=2 \mu \mathrm{A} / \mathrm{cm}^{2}\right)$.

On constate (Fig. 1) que la densité optique DO(F) au maximum de la bande $F$ est de la forme:

i) $\mathrm{DO}(\mathrm{F}) \propto t^{0,5}$ pour les cristaux de Korth ainsi que pour les cristaux de Harshaw contaminés avec diverses impuretés.

ii) $\mathrm{DO}(\mathrm{F}) \propto t^{0,75}$ pour les cristaux de Harshaw.

Ces lois sont valables dans un domaine de concentrations allant de $10^{17}$ à $10^{19}$ centres $\mathrm{F} / \mathrm{cm}^{3}$.

La figure 2 montre que, pour les cristaux de provenance Korth, la concentration en centres $F$ évolue, à temps d'irradiation fixe, comme la racine carrée de la densité d'irradiation électronique $j$, donc:

$$
\mathrm{DO}(\mathrm{F}) \propto(\dot{\varepsilon} t)^{0,5},
$$

où $\dot{\varepsilon}$ est le taux de déposition d'énergie par unité de volume proportionnel à $j$.

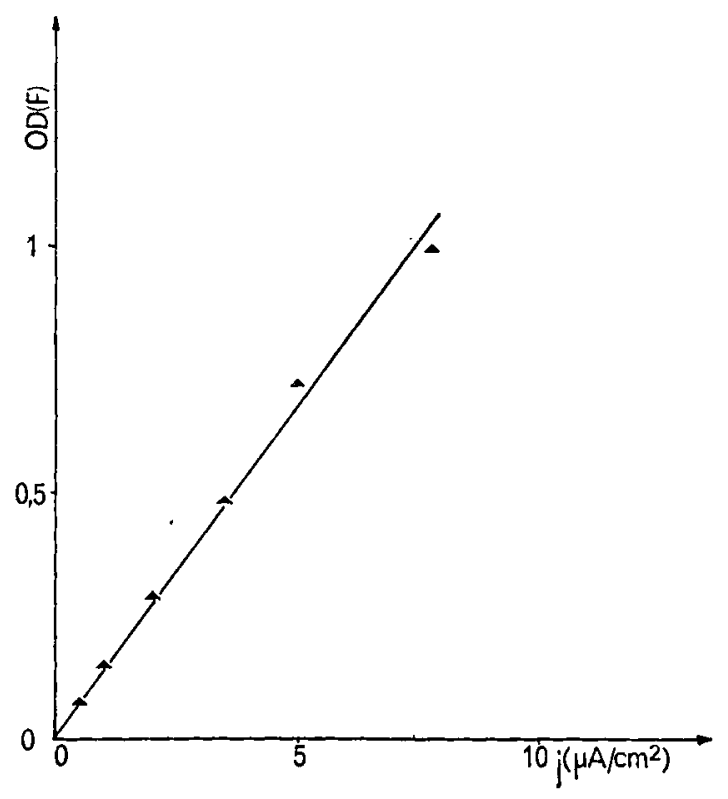

Frg. 2. - Densité optique au maximum de la bande $F$ en fonction de la racine carrée de la densité électronique $j$ pour $\mathrm{KBr}$ (Korth ultra-pur) (irradiations pendant $5 \mathrm{~min}$ à $V=20 \mathrm{kV}$, $T=80 \mathrm{~K})$.

Par contre, pour les cristaux de provenance Harshaw, la loi expérimentale en fonction de $\dot{\varepsilon}$ est de la forme (Fig. 3) :

$$
\mathrm{DO}(\mathrm{F}) \propto \dot{\varepsilon} \cdot t^{0,75}
$$

Dans ce cas, DO(F) n'est pas simplement fonction de la dose d'énergie reçue $\dot{\varepsilon} t$.

De plus, le taux de formation des centres $F$ (après $10 \mathrm{~s}$ d'irradiation) est environ trois fois plus important

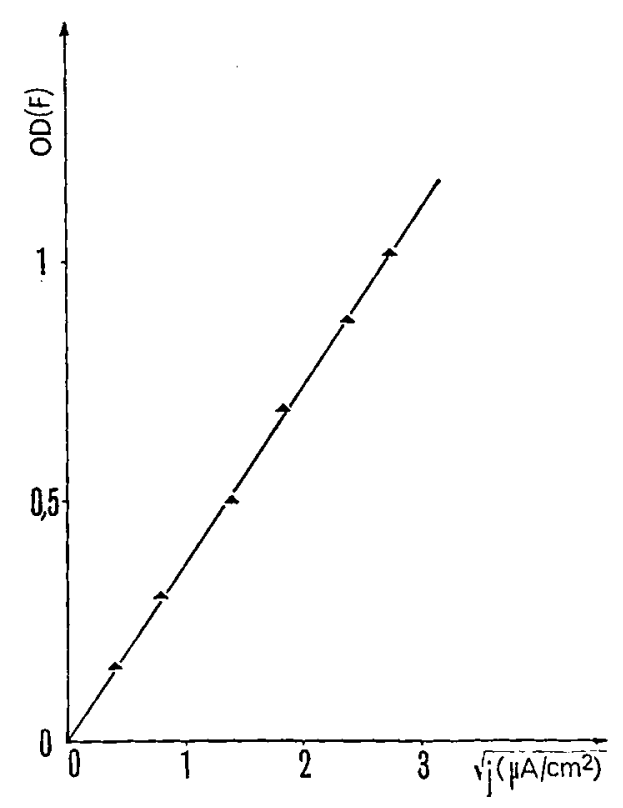

Fig. 3. - Densité optique au maximum de la bande $\mathrm{F}$ en fonction de la densité électronique $j$ pour $\mathrm{KBr}$ (Harshaw) (irradiations pendant $5 \min$ à $V=20 \mathrm{kV}, T=80 \mathrm{~K}$ ). 
dans les cristaux de provenance Korth que dans ceux de provenance Harshaw.

2. Le modèle. - Pour interpréter ces résultats, nous nous placerons dans le modèle déjà développé par plusieurs auteurs [9], [10]. L'idée principale consiste à utiliser des équations dont les variables sont des concentrations. L'emploi de telles méthodes est suggéré par leur grand succès en métallurgie [13]. $\mathrm{Ce}$ modèle est basé sur les hypothèses suivantes:

1) Le taux de création primaire des paires de Frenkel est égal à $\alpha \dot{\varepsilon}$ où le rendement de production $\propto$ n'est pas fonction du temps.

2) La probabilité de créer un centre $F$ stable est la même sur tous les sites cristallins et les deux partenaires de la paire de Frenkel sont non corrélés. Ceci justifie l'utilisation de concentrations comme variables.

3) Les interstitiels mobiles peuvent :

- soit se recombiner avec les lacunes,

— soit être piégés.

Nous pouvons alors écrire les équations suivantes:

$$
\begin{aligned}
& \frac{\mathrm{d} i}{\mathrm{~d} t}=\alpha \dot{\varepsilon}-B i l-C N i \\
& \frac{\mathrm{d} l}{\mathrm{~d} t}=\alpha \dot{\varepsilon}-B i l
\end{aligned}
$$

où $i$ et $l$ sont les concentrations en interstitiels libres et en lacunes, $N$ est la concentration des pièges à interstitiels, $B$ et $C$ caractérisent les probabilités de recombinaison et de piégeage, fonctions du coefficient de diffusion de l'interstitiel mobile et des sections de capture de la lacune et des pièges respectivement.

Compte tenu de la faible durée de vie des interstitiels [14], il suffit de considérer l'état stationnaire, c'est-à-dire $\mathrm{d} i / \mathrm{d} t \approx 0$, ce qui donne :

$$
i=\frac{\alpha \dot{\varepsilon}}{B l+C N}
$$

et

$$
\frac{\mathrm{d} l}{\mathrm{~d} t}=\alpha \dot{\varepsilon} \frac{C N}{B l+C N}
$$

On peut admettre que cette relation (4) décrit de façon correcte le taux de formation des centres $F$, ceux-ci constituant, en effet, à $80 \mathrm{~K}$, la plus grande partie des lacunes.

3. Interprétation et discussion. - La relation (4) fournit une loi de croissance en puissance de $t$, c'està-dire de la forme de celles trouvées expérimentalement si $C N$ est négligeable devant $B F$. Ceci implique que les interstitiels se stabilisent sous forme dagglomérats.

L'exposant est $\frac{1}{2}$ si $C N$ n'est pas fonction de $\mathrm{F}$, il est supérieur si $C N$ augmente avec $\mathrm{F}$.

On pourrait penser que la différence de comportement entre les cristaux purs et impurs provient de l'existence d'agglomérats de tailles différentes suivant la pureté. Si l'on raisonne qualitativement, il est logique d'admettre que, dans les cristaux qui ont assez de sites de nucléation, les agglomérats d'interstitiels sont relativement petits et qu'alors leur section de capture, pour le piégeage, est déterminée par un champ de force qui ne dépend pas de leur taille, donc $C N$ n'est pas fonction de F. Dans les cristaux plus purs, les amas d'interstitiels sont plus importants et leur taille devient très rapidement de l'ordre de grandeur du domaine d'interaction. Dans ce cas, la section de capture croît en même temps que la taille : $C N$ augmente avec $\mathrm{F}$ et on s'attend alors à avoir une loi en puissance supérieure à $\frac{1}{2}$.

Cette interprétation n'est pas valable du fait que les lois sont vérifiées sur pratiquement deux décades de concentration en centres $F$ quelle que soit la provenance des échantillons. Si l'on admet, en effet, que dans $\mathrm{KBr}$ (Korth) ils sont, en fin d'irradiation, toujours plus petits que ceux créés au début du bombardement dans $\mathrm{KBr}$ (Harshaw), on déduit alors, dans ces derniers, des dimensions pour les agglomérats peu vraisemblables (1000 à 10000 atomes d'halogènes).

Pour l'interprétation de nos résultats nous proposons alors les hypothèses suivantes:

i) Dans les cristaux impurs, le nombre des agglomérats reste constant au cours de l'irradiation et leur section de capture est indépendante de leur taille. On peut concevoir une interaction à longue portée entre le piège et l'interstitiel mobile. Dans ce cas, la loi de croissance est, d'après [4], de la forme:

$$
\mathrm{F} \propto(\dot{\varepsilon} t)^{1 / 2} .
$$

ii) Dans les cristaux purs, cette sorte de piège, caractérisée par une force à longue portée, n'existe pas. Les agglomérats d'interstitiels ont une section de capture qui est fonction de leur dimension. Ceci conduit à une loi de croissance avec une puissance comprise entre 0,5 et 1 . Par exemple, un modèle très simple d'amas plans circulaires de section de capture proportionnelle au rayon fournit une loi de la forme:

$$
\mathrm{F} \propto(\dot{\varepsilon} t)^{2 / 3} .
$$

La différence de comportement entre les cristaux purs et impurs ne serait donc pas due à une différence de taille des agglomérats d'interstitiels mais de la nature de l'interaction interstitiel-piège. 


\section{Bibliographie}

[1] Crawford, J. H. Jr., Adv. Phys, 17 (1968) 93.

[2] Pooley, D., Proc. Phys. Soc. 87 (1966) 245 ; Proc. Phys. Soc. 87 (1966) 257.

[3] Sonder, E., Sibley, W. A., Phys. Rev. 140 (1965) A 538.

[4] Sonder, E., Bassignani, G., Camagni, P., Phys. Rev. 180 (1969) 882.

[5] Garth, J. C., Sonder, E., J. Phys. \& Chem. Solids 29 (1968) 1737.

[6] Sonder, E., Walton, D., Phys. Lett. 25A (1967) 222.

[7] Guenther, R. A., Weinstock, H., J. Appl. Phys, 42 (1971) 3790.
[8] Hobbs, L. W., Hughes, A. E., Pooley, D., Proc. R. Soc. Lond. A 332 (1973) 167.

[9] Sonder, E., Phys. Stat. Sol. 35 (1969) 523.

[10] Durand, P., Farge, Y., Lambert, M., J. Phys. \& Chem. Solids 30 (1969) 1353.

[11] Sonder, E., Sibley, W. A., Point Defects in Solids, ed. by J. H. Crawford and L. F. Slifkin (Plenum Press, New York) 1972.

[12] Alvarez Rivas, J. L., Solid State Commun. 9 (1971) 1025.

[13] Walker, R. M., Radiation Damage in Solids, ed. by D. S. Billington (Academic Press) 1962.

[14] Ueta, M., J. Phys. Soc. Japan 23 (1967) 1265.

\section{DISCUSSION}

L. W. HoBbs. - You must be careful to distinguish between the size of an interstitial cluster and the extent of its strain field when you speak of the cross section for interstitial trapping. It is the latter which is responsible for the elastic interaction between an $\mathrm{H}$ centre and an interstitial cluster. The extent of the strain field will depend to some extent on the size of the cluster, but also critically on the cluster morphology, e. g. whether the clusters are essentially one or two dimensional, etc. In any event, the extent of the strain field can be considerably longer range than the actual size of the cluster. Since it has been shown that interstitial anions in alkali halides stabilize in perfect dislocations loopsof either round or dipole form, the usual formulations for the strain fields of dislocation loops or dipoles can be used to estimate the extent of the $\mathrm{H}$ centre-interstitial cluster interaction.

A. E. Hughes. - Your models depend very heavily on the experimental power laws. Those have been obtained assuming average values for the radia- tion intensity over the irradiated volume of the crystal. In fact, the intensity varies considerably over this volume owing to the non-uniform energy loss of the electrons. Have you taken this into account?

P. PINARD. - It is sure that $\dot{\varepsilon}$ the energy deposition rate is not constant over the volume. One could think that the experimental curve $\mathrm{OD}(t)$ (that is the integral growth curve) is then the result of the superposition of an infinity of local growth curves $\alpha(t, x)$ and one could wait for a difficult interpretation of $\mathrm{OD}(t)$. We have shown that the form of the coloration profile has very little influence on the integral growth curve by choosing two extreme cases for $\alpha(t, x)$ first : all the electrons have the same energy at a certain depth, second: complete dispersion is assumed and the energy deposition rate is independent of $x$. The two functions $\alpha_{1}$ and $\alpha_{2}$ found are very close together the maximum deviation being $4 \%$. The curves $\alpha(t)$ and $\mathrm{DO}(t)$ can thus be identified without much error. 